\title{
Giant Fluctuations Induced by Thermal Diffusion in Complex Liquids
}

\author{
Alberto Vailati ${ }^{1}$ (D) Philipp Baaske ${ }^{2} \cdot$ Henri Bataller $^{3} \cdot$ Serena Bolis ${ }^{4} \cdot$ Marco Braibanti $^{5} \cdot$ Marina Carpineti $^{1}$. \\ Roberto Cerbino $^{6}$ - Fabrizio Croccolo ${ }^{3}$. Jean-Luc Dewandel ${ }^{4}$ - Aleksandar Donev ${ }^{7}$ - Loreto García-Fernández ${ }^{3,13}$. \\ Fabio Giavazzi ${ }^{6}$ - Robert Haslinger ${ }^{2}$. Steven Hens ${ }^{8}$ - Maria Knauer ${ }^{2}$ • Werner Köhler ${ }^{9}$ • Ewald Kufner ${ }^{10}$ • José M. Ortiz \\ de Zárate ${ }^{11} \cdot$ Jeroen Peeters ${ }^{8} \cdot$ Christian J. Schwarz $^{10} \cdot$ Inese Silkina $^{10} \cdot$ Shenghua Xu $^{12} \cdot$ Daniel Zapf $^{9}$
}

Received: 30 March 2020 / Accepted: 28 June 2020 / Published online: 14 July 2020

(C) The Author(s) 2020

\begin{abstract}
The GRADFLEX experiment of ESA has shown that under microgravity conditions a stationary thermodiffusion process is accompanied by giant non-equilibrium fluctuations with size as large as the size of the sample. In the presence of small concentration gradients, the features of the non-equilibrium fluctuations can be described by means of linearized hydrodynamics. However, the linear models are not suitable to describe most cases of applicative interest, such as fluctuations induced by large gradients and under non-stationary conditions. Moreover, presently the investigation of non-equilibrium fluctuations has mainly involved single component fluids and binary mixtures, but recently transport processes in ternary mixtures have attracted increasing interest due to the experiments performed on the International Space Station in the framework of the DCMIX project of ESA. The Giant Fluctuations (NEUF-DIX) project of ESA will investigate non-equilibrium fluctuations during diffusive processes occurring in complex multi-component mixtures, where one of the components is a macromolecule, such as a polymer, a colloid or a protein. Important objectives will be the exploration of the features of the fluctuations under non-ideal conditions, such as large gradients, transient processes, and concentrated samples, and the understanding of how the fluctuations affect the interactions between macromolecules. The project involves the development of a dedicated facility, consisting of an array of shadowgraph optical instruments working in parallel, each one equipped with a thermal gradient cell. Here we outline the design concept of the facility and the results of performance tests performed on a breadboard to evaluate the suitability of the designed instrument to carry out scientific measurements of non-equilibrium concentration fluctuations in space.
\end{abstract}

Keywords Thermodiffusion $\cdot$ Microgravity $\cdot$ Non-equilibrium fluctuations $\cdot$ Shadowgraph $\cdot$ Transport properties

This article belongs to the Topical Collection: The Effect of Gravity on Physical and Biological Phenomena

Guest Editor: Valentina Shevtsova

Alberto Vailati

alberto.vailati@unimi.it

1 Dipartimento di Fisica “A. Pontremoli”, Università degli Studi di Milano, Milan, Italy

2 NanoTemper Technologies GmbH, Munich, Germany

3 Universite de Pau et des Pays de l'Adour, E2S UPPA, CNRS, TOTAL, LFCR UMR5150, Anglet, France

4 Lambda-X, Nivelles, Belgium

5 HE Space Operations BV for ESA, NL-2200AG Noordwijk, The Netherlands

6 Dipartimento di Biotecnologie Mediche e Medicina Traslazionale, Università degli Studi di Milano, Segrate, MI, Italy
7 Courant Institute of Mathematical Sciences, New York University, New York, NY, USA

8 Qinetiq Space, Kruibeke, Belgium

9 Physikalisches Institut, Universität Bayreuth, Bayreuth, Germany

10 ESA-ESTEC, Noordwijk, The Netherlands

11 Departamento de Estructura de la Materia, Física Térmica y Electrónica, Facultad de Ciencias Físicas, Universidad Complutense de Madrid, Madrid, Spain

12 Institute of Mechanics, Chinese Academy of Sciences, Beijing, China

13 Centre National d'Études Spatiales, Paris, France 


\section{Introduction}

In the presence of a macroscopic concentration gradient, a fluid mixture exhibits non-equilibrium fluctuations determined by the coupling of the gradient to equilibrium velocity fluctuations (Ortiz de Zárate and Sengers 2006). The amplitude of non-equilibrium concentrations fluctuations can become orders of magnitude larger than that of equilibrium ones. This feature is apparent from the structure factor of the fluctuations, which exhibits a power-law divergence $S(q) \propto q^{-4}$, where $q$ is the wave number. This power law behavior is the signature of a scale invariant behavior, which in the case of non-equilibrium fluctuations is reflected by the self-affine structure of the fronts of diffusion (Brogioli et al. 2016; Brogioli and Vailati 2017). Theoretical predictions have shown that on Earth the divergence of the structure factor of non-equilibrium fluctuations is prevented by the gravity force (Segrè and Sengers 1993). This result has been confirmed experimentally by using small-angle light scattering (Vailati and Giglio 1996, 1997). The investigation of the dynamics of relaxation of the fluctuations showed that on Earth nonequilibrium fluctuations relax diffusively, while at small wave vector their relaxation is determined by buoyancy (Croccolo et al. 2006). At very small wave vectors the effect of buoyancy becomes strong and gives rise to propagating fluctuations (Croccolo et al. 2019).

In the absence of gravity, the divergence of the amplitude of non-equilibrium fluctuations at small wave vectors is prevented by the finite thickness of the fluid layer (Ortiz de Zárate et al. 2006). The role of the finite size of the sample was demonstrated experimentally in the framework of the GRADFLEX experiment of the European Space Agency (Vailati et al. 2006; 2011; Takacs et al. 2011; Cerbino et al. 2015; Croccolo et al. 2016). GRADFLEX flew on FOTON M3 in 2007 and provided quantitative evidence that in the absence of gravity the static structure factor diverges as $q^{-4}$, for wave vectors larger than the wave vector $\pi / h$ associated to the finite thickness of the sample $h$, below which the structure factor saturates to a constant value (Vailati et al. 2011). As far as the dynamics of fluctuations is involved, GRADFLEX showed that in the absence of gravity the relaxation of fluctuations is purely diffusive, irrespective of the wave vector. Further experiments performed on Earth investigated the combined action of gravity and finite size on the slowing down of non-equilibrium fluctuations (Giraudet et al. 2015).

The results obtained with GRADFLEX allowed to establish that linearized hydrodynamics is suitable to provide a fully quantitative description of the static structure factor of non-equilibrium fluctuations under the ideal conditions where the amplitude of fluctuations is small, and the diffusive process is stationary. At the same time, the development of the DCMIX project of the European Space Agency showed the interest of a wide community of scientists in the investigation of mass transport processes in multi-component liquid mixtures (Bou-Ali et al. 2015; Galand et al. 2019; Shevtsova et al. 2014; Triller et al. 2018; Mialdun et al. 2019). Multi component mixtures exhibit a rich stability diagram, where the stability of the sample in the presence of gravity is compromised by the presence of double diffusion processes, which can lead to convective instabilities even when the initial density profile of the sample is stable (Šeta et al. 2019). For these reasons, the main objective of DCMIX has been the investigation of the macroscopic mass transport process in ternary mixtures in the absence of gravity, so to prevent the onset of convective instabilities.

The aim of the "Giant Fluctuations" (NEUF-DIX) project of ESA is to take advantage of the experience gathered with GRADFLEX and DCMIX and investigate non-equilibrium fluctuations occurring during thermodiffusion in multicomponent mixtures under non-ideal conditions of interest for practical applications (Baaske et al. 2016). These nonideal conditions include the investigation of large amplitude fluctuations induced by large gradients, the investigation of transient diffusion processes, which are currently poorly understood theoretically, and the investigation of the role of nonequilibrium fluctuations in the interaction between macromolecules, which can be affected by thermal forces determined by the confinement of non-equilibrium fluctuations (Kirkpatrick et al. 2013, 2015). The project will focus on the investigation of non-equilibrium fluctuations in complex fluids, such as multicomponent mixtures including a polymer as one of the components, colloidal suspensions and protein solutions. The project has now finished the $\mathrm{A} / \mathrm{B}$ phase and is scheduled for flight in 2022. The preliminary design of the flight hardware comprises five instruments working in parallel, each one composed by two-color shadowgraph optical diagnostics and a thermal gradient cell. We report below the current status of the development of the flight hardware and the test measurements that have been performed to evaluate the performances of the instrument by using samples similar to those that will be used for space experiments.

\section{Theoretical Background}

The investigation of non-equilibrium fluctuations requires creating a macroscopic concentration gradient in a mixture of fluids. On Earth this can be performed under isothermal conditions by layering two miscible phases, one on top of the other. By putting the denser phase below and the lighter one above the system is gravitationally stable and remixes through an isothermal diffusion process (Vailati and Giglio 1998; Brogioli et al. 2000; Croccolo et al. 2006; Giavazzi et al. 2016a, 2016b). Under microgravity conditions, fluid phases cannot be layered with the help of gravity, but one can take advantage of thermodiffusion, which induces a concentration gradient after imposing a temperature gradient to a mixture. 


\section{Thermodiffusion}

When a temperature gradient is applied to a multi-component mixture, the components undergo a partial separation. This phenomenon is known as Soret effect or thermodiffusion (de Groot and Mazur 1984). At steady state, the mass flow determined by thermodiffusion is balanced by Fickean diffusion. The result of this balance is a stationary concentration gradient inside the fluid, which is proportional to the imposed temperature gradient, $\nabla c=-S_{T} c_{0}\left(1-c_{0}\right) \nabla T$. Here $S_{T}$ is the Soret coefficient, and $c_{0}$ is the concentration of the mixture at equilibrium. Under microgravity conditions the Soret effect can be used to generate a concentration gradient in a fluid mixture under controlled conditions by applying a temperature gradient to the layer of mixture, with the advantage that experiments can be iterated at will by imposing/removing the thermal gradient. This method has been exploited during several experiments of the European Space Agency on thermodiffusion under microgravity conditions (Braibanti et al. 2019), which benefited from the fact that the absence of gravity allows to investigate purely thermodiffusive processes, in the absence of convective instabilities.

\section{Non-equilibrium Fluctuations}

The theory of non-equilibrium fluctuations is based on linearized hydrodynamics, with the addition of stochastic fluxes that act as thermal sources for the fluctuations. The derivation of these Langevin equations from fluctuating hydrodynamics is described in great detail in (Ortiz de Zárate and Sengers 2006). Here we summarize the theoretical results for the static and dynamic power spectra of non-equilibrium concentration fluctuations determined by a concentration gradient. In particular, we focus on recent theoretical developments that include realistic boundary conditions in the case when gravity is not present (Ortiz de Zárate et al. 2015), as it occurs for experiments performed in space. Under these conditions the dynamic structure factor of concentration non-equilibrium fluctuations is:

$S(\omega, q)=\frac{k_{B} T(\nabla c)^{2}}{\rho v D q^{4}} \frac{2 D q^{2}}{\omega^{2}+D^{2} q^{4}}\left[1+\frac{4(1-\cosh \tilde{q})}{\tilde{q}(\tilde{q}+\sinh \tilde{q})}\right]$

where $k_{B}$ is the Boltzmann constant, $T$ the average temperature, $\tilde{q}=q h$ the dimensionless wave number, $\rho$ the mass density, $\omega$ the frequency, $v$ the kinematic viscosity, and D the diffusion coefficient.

The terms of Eq. 1 outside square brackets show that the power spectrum has a Lorentzian shape, with an amplitude diverging as $q^{-4}$ at small wave vectors. The factor between square brackets takes into account the finite size of the sample and exhibits a saturation to a constant value below $\tilde{q}=\pi$. The relaxation time of the fluctuations is inversely proportional to the width of the Lorentzian spectrum in Eq. 1:

$\tau(q)=\frac{1}{D q^{2}}$

The static structure factor of the fluctuations can be obtained by integrating Eq. 1 across the frequency domain:

$S_{c c}^{N E \infty}=\frac{k_{B} T(\nabla c)^{2}}{\rho v D q^{4}}\left[1+\frac{4(1-\cosh \tilde{q})}{\tilde{q}(\tilde{q}+\sinh \tilde{q})}\right]$

The theoretical results in Eqs. 2 and 3 have been confirmed experimentally during the GRADFLEX experiment, (Vailati et al. 2011; Cerbino et al. 2015; Croccolo et al. 2016). In GRADFLEX, non-equilibrium concentration fluctuations were induced through the Soret effect by imposing a temperature difference to a polymer solution of polystyrene with a molecular weight of $9100 \mathrm{~g} / \mathrm{mol}$ at a weak concentration of $1.8 \% \mathrm{w} / \mathrm{w}$ dissolved in toluene. Experiments were performed under ideal conditions suitable for a fully quantitative comparison with the theoretical predictions, namely, diluted samples and stationary conditions.

\section{The Giant-Fluctuations Project (NEUF-DIX)}

The Giant Fluctuations project of the European Space Agency is aimed at the investigation of Non-EqUilibrium Fluctuations occurring during DIffusion processes in multi-component compleX fluids (NEUF-DIX) in the absence of gravity, under conditions that cannot be easily tackled by theoretical models (Fig. 1).

These include transient diffusion, concentrated samples and large gradients, also in comparison to computational results, by taking advantage of the computational methods recently developed for the investigation of multiscale problems (Donev et al. 2011; Balboa Usabiaga et al. 2012; Delong et al. 2014; Donev et al. 2014a). The focus of the project is on complex liquids, because of the rich phenomenology exhibited by such systems. A preliminary selection of samples that will be investigated during the first flight campaign is detailed in Table 1. Part of the project is also devoted to the development of diagnostic tools based on non-equilibrium fluctuations, in the framework of the CORA-MAP project "Technologies for NonEquilibrium Systems" (TechNES). The main scientific goals of the project are represented by the investigation of several outstanding scientific problems, outlined in the following. 
Fig. 1 Overall plan of fundamental and applicative objectives of the Giant Fluctuations project

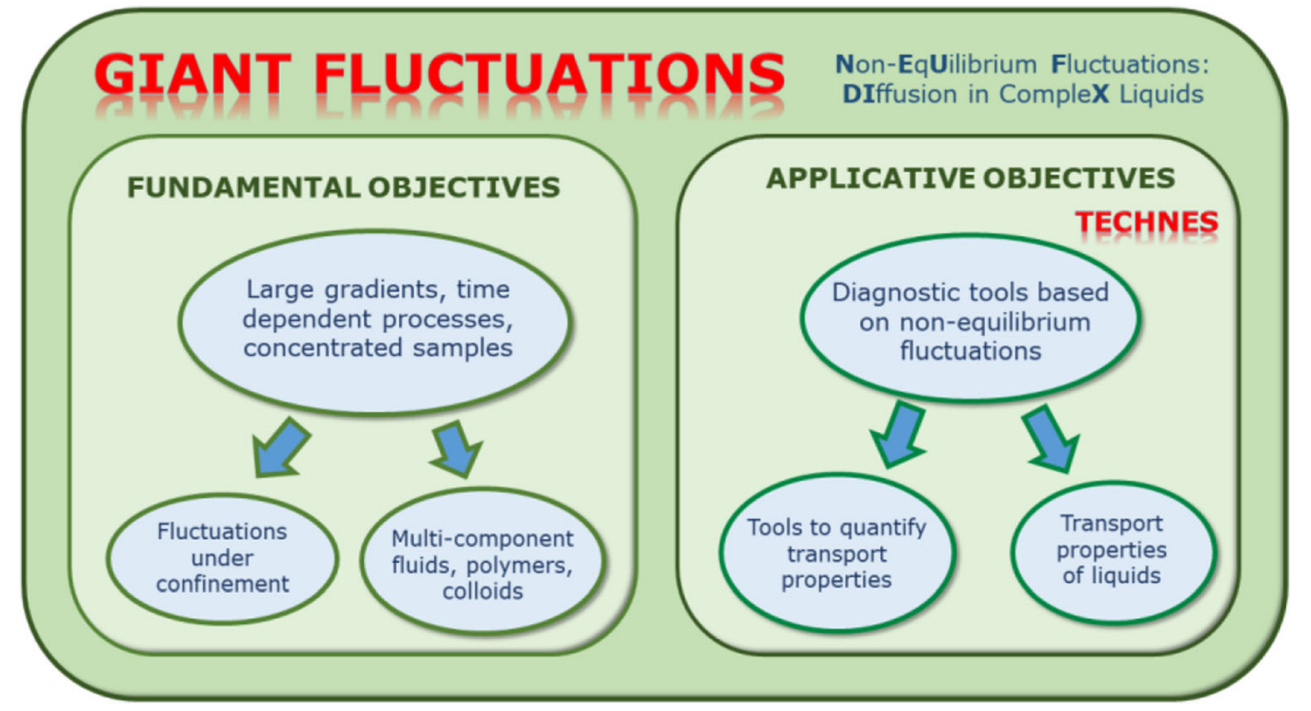

\section{Non-equilibrium Fluctuations in a Complex Mixture Including a Polymer}

The investigation of non-equilibrium fluctuations allows to access different physical processes taking place during diffusion (mass diffusion, Soret and thermal diffusivity coefficients, viscosity (Croccolo et al. 2012; Giraudet et al. 2014; Lizarraga et al. 2016; Bataller et al. 2016, 2017). Microgravity conditions are mandatory for the measurement of transport coefficients, because they suppress convective motions associated to temperature and concentration gradients. The ideal mixture to perform such an investigation is a ternary mixture where one of the components has a much larger molecular weight. Under these conditions, the two eigenvalues of the mass diffusion coefficient matrix are well separated and they can be identified accurately during experiments. This kind of system would also be ideal to verify experimentally the asymmetry of the time cross-correlation function of nonequilibrium concentration fluctuations in a ternary mixture, recently predicted theoretically (Brogioli et al. 2019).

\section{Glass Transition in a Ternary Mixture Including a Polymer}

One goal of the Giant Fluctuations project is to investigate how glass transition and chain entanglement - two different mechanisms that slow down dynamics in a concentrated polymer solution - affect the non-equilibrium temperature and concentration fluctuations. When the glass transition is approached in a supercooled liquid, the slowing down of fluctuations affects both the isothermal Fickian and thermodiffusion coefficients. However, local friction cancels out in the Soret coefficient, which scales as predicted by the blob model (Rauch and Köhler 2002, 2003). The slowingdown of diffusion close to the glass transition is remarkably different from the critical slowing-down near a consolute critical point, where the Soret coefficient diverges whereas the thermodiffusion coefficient remains unaffected (Enge and Köhler 2004). On Earth, the combination of gravity and confinement determines an additional slowing-down of the nonequilibrium fluctuations (Giraudet et al. 2015), making the effect of approaching the glass transition difficult to evaluate. Conversely, the observation of non-equilibrium fluctuations in microgravity represents a good method to quantify diffusion coefficients (Vailati et al. 2011), and one expects it to be a suitable tool to observe the slowing down when the glass transition is approached. A polymer such as polystyrene in a (mixed) organic solvent is ideally suited for these investigations. It represents not only a highly asymmetric system with small and large molecules but also a mixture with a high $T_{g}$ contrast of its constituents, where the distance to the glass transition temperature $T_{g}$ can be adjusted by both temperature and composition variations.
Table 1 Liquid mixtures selected for the first flight campaign and their composition

\begin{tabular}{lll}
\hline Experiment & Sample & Concentration w/w \\
\hline Ternary mixtures & Toluene+Cyclohexane+Polystyrene Polymer & $39-59-2 \%, 4730 \mathrm{~g} / \mathrm{mol}$ \\
Glass transitions & Toluene+Cyclohexane+Polystyrene Polymer & $36-35-25 \% 5000 \mathrm{~g} / \mathrm{mol}$ \\
Colloids & Ludox in water & $34-66 \%$ \\
Transients & Toluene+ Polystyrene Polymer & $98-2 \% 9000 \mathrm{~g} / \mathrm{mol}$ \\
Biological samples & Hen egg white Lysozyme in water & TBD \\
\hline
\end{tabular}




\section{Casimir Forces in Equilibrium and Out of Equilibrium}

Long-ranged fluctuations are affected by the presence of boundaries and, in turn, the presence of long-ranged fluctuations affects the boundaries, inducing forces on them. In the case of a liquid mixture at thermodynamic equilibrium, fluctuations are only long-ranged close to the critical point. Recent theoretical work predicted the existence of a, yet unobserved, non-equilibrium Casimir effect determined by the confinement of nonequilibrium fluctuations, that would produce forces much larger than those reported for the equilibrium critical Casimir effect (Kirkpatrick et al. 2013, 2015). The most suitable method for detecting the presence of forces induced by non-equilibrium fluctuations makes use of colloidal particles to confine the fluctuations. Under these conditions, the forces generated by the confinements should affect the interactions of the colloidal particles, leading to the formation of clusters of particles.

\section{Transient Fluctuations}

Non-equilibrium fluctuations have been investigated mostly at steady-state or during quasi-stationary diffusion processes (Vailati and Giglio 1997, 1998; Brogioli et al. 2000; Croccolo et al. 2007; Giavazzi et al. 2016a). An open question is represented by the kinetics of growth of fluctuations during the development of a macroscopic concentration gradient in a multi-component mixture. On Earth, the investigation of the kinetics of growth of the fluctuations is strongly affected by the gravitational force, and the growth rate of the static structure factor of non-equilibrium fluctuations appears to be independent from the wave vector (Carpineti et al. 2019). Recent simulations investigated the growth of non-equilibrium fluctuations induced by the Soret effect in a solution of a polystyrene polymer in toluene under conditions mirroring those of the GRADFLEX experiment (Cerbino et al. 2015). The simulations show the development of a transient peak of the structure factor, gradually moving at small wave vectors. The comparison with experimental results shows that simulations agree only partially with the data collected during GRADFLEX, the lack of agreement being mostly due to the poor statistical sample available for the experimental data collected during the space experiment. For this reason, future experiments on transient fluctuations will be performed under conditions that allow collecting a larger statistical sample. The improvement of the statistical sample can be achieved both by iterating the experiment several times, and by using a mixture where one of the components is made of macromolecules, to slow down the kinetics of growth of the fluctuations.

\section{Dense Colloidal Suspensions}

So far, the investigation of non-equilibrium fluctuations in complex fluids has been focused mostly onto polymer suspensions (Vailati et al. 2011; Cerbino et al. 2015; Croccolo et al. 2016) and dilute colloidal suspensions (Croccolo et al. 2006; Oprisan et al. 2010). Only recently experiments on concentrated colloidal suspensions have been performed on Earth (Giavazzi et al. 2016a), but the only available theoretical model for non-equilibrium concentration fluctuations in concentrated colloids (Schmitz 1994) is rather dated and not able to capture the phenomenology exhibited by experimental results. Alternative theoretical approaches, as for instance Dynamic Density Functional Theory, have been recently used to tackle the problem (Donev and Vanden-Eijnden 2014b), and much can be done both from the experimental and the theoretical side to investigate fluctuations in dense colloidal suspensions, a promising and almost unexplored, experimental field.

\section{Biological Samples}

The development of proteinaceous drugs represents an important goal of the pharmaceutical industry, because they have higher specificity and effectiveness, with fewer side effects than conventional chemical drugs. However, these proteinaceous drugs introduce new challenges, because of unwanted effects like the self-association of proteins or their aggregation. Thermal diffusion methods are already established to study biomolecular solutions, but the microscale thermophoresis signal is often strongly influenced by thermal convection. Microgravity conditions are thus essential for the measurement of the microscale thermophoresis of biomolecules. Moreover, the confinement of long-range fluctuations determined by proteins in space is expected to induce forces between them contributing to their aggregation. Space experiments will provide an insight to better understand the mechanisms that induce aggregation of proteins under nonequilibrium conditions.

\section{Experimental Breadboard}

The investigation of all the topics detailed above requires the availability of a thermal gradient cell in order to impose a controlled temperature difference to a layer of sample, sided by a quantitative optical diagnostics method able to discriminate the small optical signal generated by non-equilibrium fluctuations during thermodiffusion in a multi-component mixture. GRADFLEX showed that shadowgraphy represents a simple and rugged optical method suitable for a fully quantitative characterization of the structure factor of nonequilibrium fluctuations under microgravity conditions. More in detail, shadowgraph has a sensitivity to small phase perturbations much larger than that of light scattering in the far field (Cerbino and Vailati 2009) and, in contrast to Small Angle Light Scattering, allows to access ultra-low wave vectors without the need of an accurate alignment. Moreover, 
shadowgraph allows performing absolute measurements of the scattering amplitude without the need of calibration measurements.

At the same time, GRADFLEX introduced a new design concept for thermal gradient cells, using sapphire windows coupled to annular Peltier elements to guarantee optical access along the direction of the imposed gradient, a condition needed for the detection of non-equilibrium fluctuations. The experience gathered with GRADFLEX allowed to conceive an experimental unit comprising multiple shadowgraph setups, working in parallel to maximize the scientific return of a space mission. An overall view of the experimental unit concept is shown in Fig. 2.

Each shadowgraph diagnostics comprises one red and one violet Superluminous Light Emitting Diode (SLED) operating at $405 \mathrm{~nm}$ and $650 \mathrm{~nm}$, whose light gets combined and collimated onto the sample cell. The choice of two separate light sources is dictated by the fact that this solution allows the discrimination of the contributions of fluctuations of the index of refraction determined by fluctuations of the concentration of the components of a ternary mixture. The decoupling of these kind of contributions by using two light sources has been pioneered during the DCMIX project, where a Mach-Zehnder interferometer working with two laser sources operating at $670 \mathrm{~nm}$ (Red) and $935 \mathrm{~nm}$ (NIR) allowed to decouple the contributions of the two independent components of a ternary mixture to the macroscopic density profile generated by a thermodiffusion process (Shevtsova et al. 2011). Multiple cells are hosted into a swappable Cell Module that allows to reuse the optical diagnostics to perform measurements with other samples.

The design concept of the cell is shown in Fig. 3. Each cell is made by two stacked sapphire windows, separated by calibrated $1 \mathrm{~mm}$ thick zirconium spacers. The sample fluid is hosted between the windows and confined laterally by a rubber gasket.

Each sapphire window is in thermal contact with an annular Peltier element, which transports heat inside a tin-bronze housing. The temperature of each window is controlled independently by using an annular Peltier Element governed by a Proportional Integral Derivative (PID) servo loop. The annular Peltier elements have a central aperture with a diameter of $27 \mathrm{~mm}$, which guarantees optical access along the direction parallel to the temperature gradient. Two thin film thermistors located at the sides of the sapphire windows in contact with the sample guarantee an accurate reading of the actual temperature difference imposed to the layer of fluid.

In order to test the suitability of this design for a space experiment on non-equilibrium fluctuations, a test breadboard was designed and built by Qinetiq Space and Lambda-X (Fig. 4). The breadboard comprises two SLED sources, one with a wavelength of $405 \mathrm{~nm}$ and the other of $650 \mathrm{~nm}$. The two sources can be turned on and off by means of an optical switch. The light emitted by them is collimated by a collimator assembly and impinges on the sample cell, where light is scattered by temperature and concentration fluctuations. A lens placed after the cell images a plane at a distance of $36 \mathrm{~cm}$ from the cell onto the sensor of a CCD camera, which collects the shadowgraph images of the fluctuations. A personal computer connected to the camera collects the shadowgraph images and stores them. To perform test measurement, the breadboard was rigged vertically and the cell was accurately levelled to a precision of 2 millirad to keep the layer of sample horizontal. During measurements, temperature differences were applied to the sample by heating from above, to avoid the onset of thermal convection during the scientific
Fig. 2 Design concept of the Giant Fluctuations experimental unit. The unit comprises five identical shadowgraph instruments and a swappable cell module. The choice of a multiple optical diagnostics allows to avoid moving parts, with the advantage of largely improving the optical alignment of parts

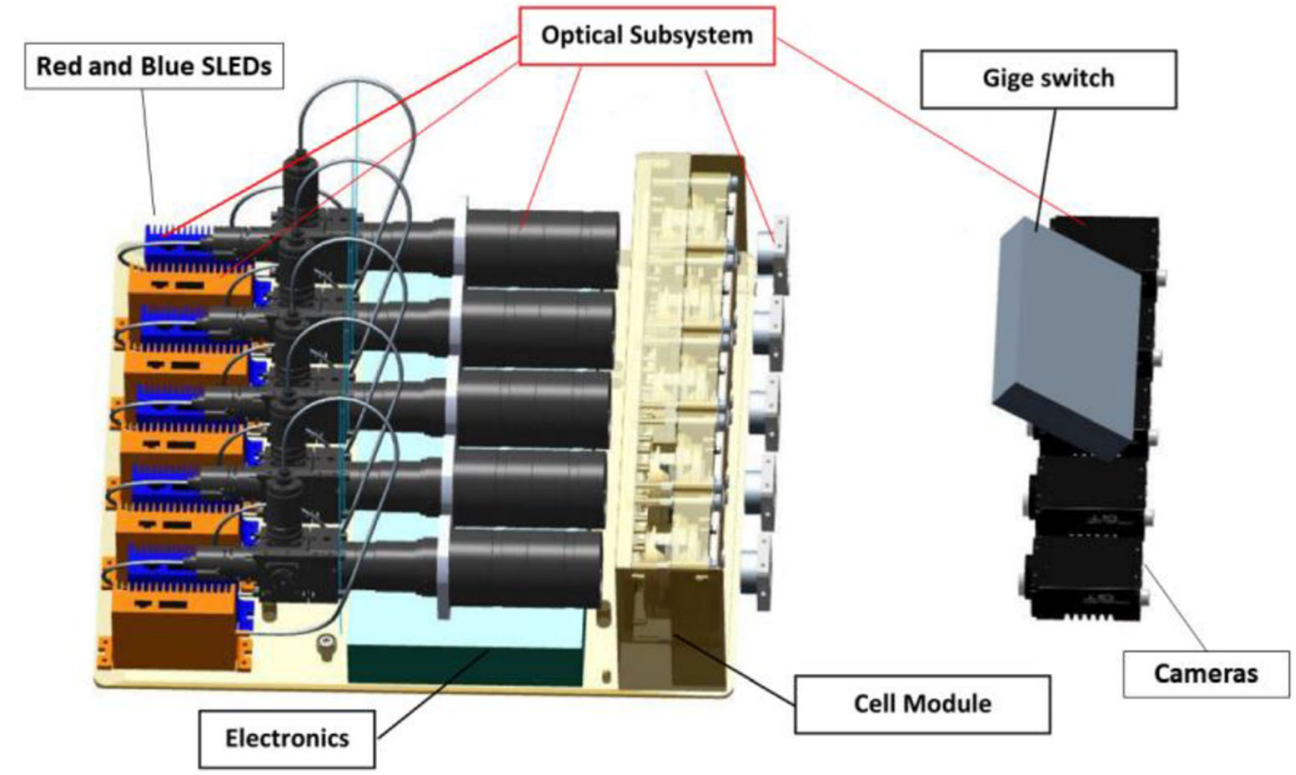


Fig. 3 Design concept of the cell breadboard. Two sapphire windows, in thermal contact with annular Peltier Elements, act as the same time as thermalizing plates and as optical observation windows

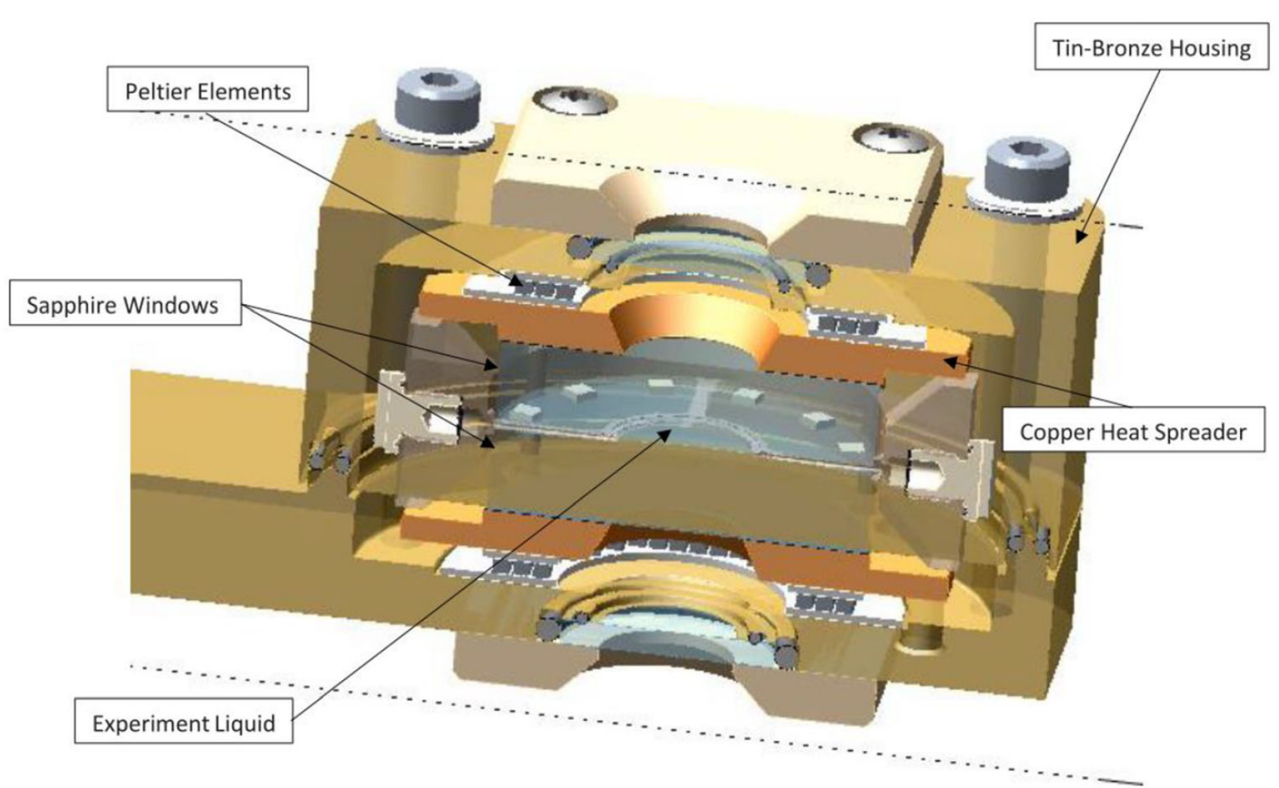

campaign on earth. In multicomponent systems there is still the possibility of the development of solutal convection, even when the sample is heated from above, which cannot be avoided in samples with unfavorable Soret coefficients. For this reason, experiments on ternary mixtures require microgravity conditions.

\section{Methods}

The general scheme for the measurement of the static and dynamic structure factors of non-equilibrium fluctuations comprises a first phase where a sequence of shadowgraph images of the sample kept at a uniform temperature is collected, and a second phase, where a temperature difference is applied and shadowgraph images are collected after a steady concentration profile has developed inside the sample. Further details about the experimental conditions chosen to perform tests will be provided below. Sequences of images have been analysed by means of the Differential Dynamic Algorithm that is able to extract the intermediate scattering function (ISF) by analysing differences of images with increasing time delay.

The algorithm was introduced in 2006 for the analysis of Shadowgraph data during diffusion experiments in binary

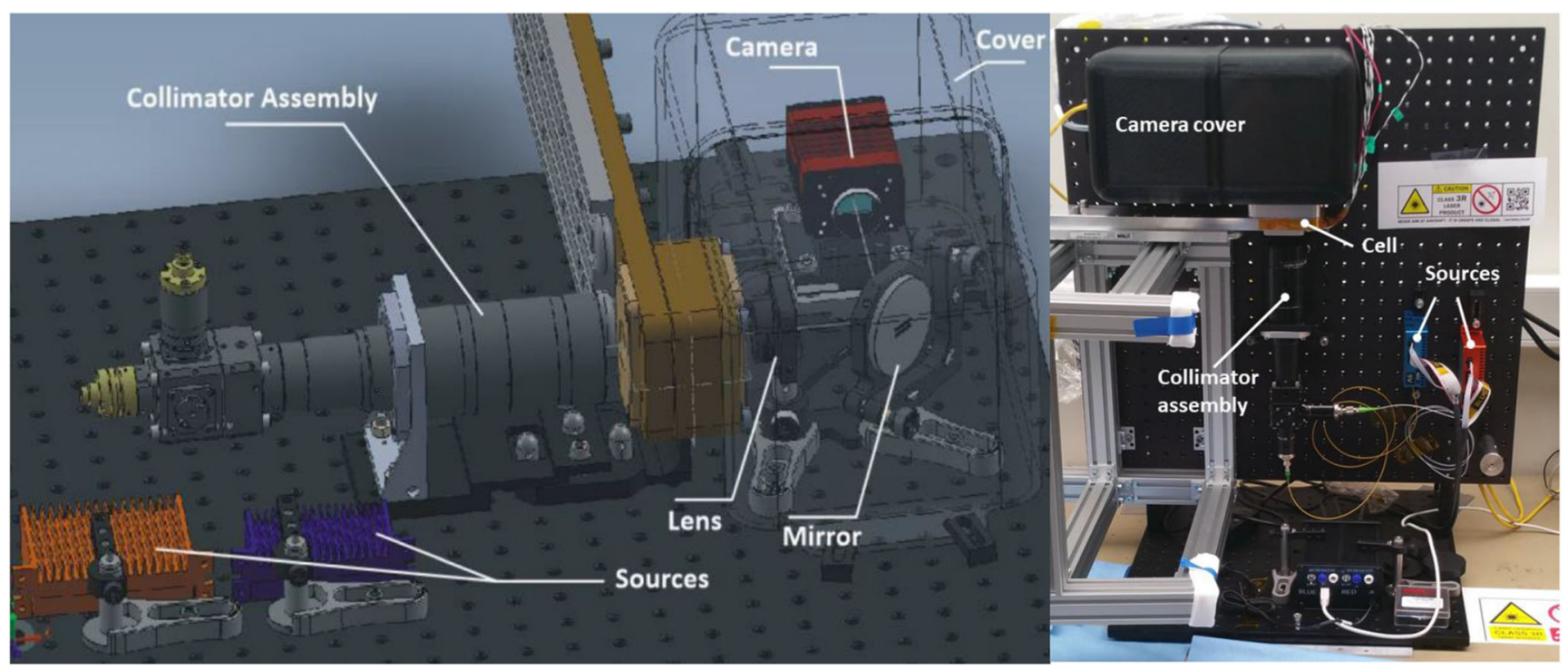

Fig. 4 Left: Design concept of the test breadboard. The light from one red and one violet Superluminous LED sources is collimated by a collimator assembly onto the cell module. Shadowgraph images of fluctuations of the index of refraction occurring inside the sample are recorded by a Charged Coupled Device sensor. Right: picture of the breadboard rigged vertically during the tests to avoid the onset of convection inside the sample 
mixtures performed on Earth under isothermal conditions (Croccolo et al. 2006, 2007). The same algorithm is adopted in the Differential Dynamic Microscopy technique, which employs an optical microscope with white light illumination to characterize the static and dynamic structure factor of a complex liquid (Cerbino and Trappe 2008; Cerbino and Vailati 2009; Giavazzi and Cerbino 2014). The Differential Dynamic Algorithm relies on the determination of the structure function of the fluctuations of the image intensity:

$C_{m}(\vec{q}, \Delta t)=\left\langle|I(\vec{q}, t+\Delta t)-I(\vec{q}, t)|^{2}\right\rangle_{t}$

where $I(\vec{q}, t)$ is the intensity distribution of a shadowgraph image in reciprocal space, and $\Delta t$ the temporal delay between two images.

In the presence of an isotropic process, it can be shown that the azimuthally-averaged structure function can be written in the general form:

$C_{m}(q, \Delta t)=2\{S(q) \cdot T(q)[1-D(q, \Delta t)]+B(q)\}$

where $T(q)$ is the optical transfer function of shadowgraph (Trainoff and Cannell 2002; Croccolo and Brogioli 2011), $S(q)$ is the static power spectrum of the concentration fluctuations, and $B(q)$ is a background contribution determined by the camera noise and by equilibrium fluctuations (Cerbino et al. 2015).

The Intermediate Scattering Function (ISF) of the system can be written under generic conditions as:

$D(q, \Delta t)=\exp \left[-\frac{\Delta t}{\tau(q)}\right]$

The fitting of experimental structure function through Eqs. 5 and 6 allows to determine the relaxation time of fluctuations. In the case of a microgravity experiment recent theories confirm the prediction of a pure diffusive behavior of concentration non-equilibrium fluctuations, even in the presence of nonnegligible confinement effects, so that the time decay is expected to be described by Eq. 1 (Ortiz de Zarate et al. 2015). A fitting of the experimental data of $\tau(q)$ as a function of the wave number can thus provide a quantitative measurement of the mass diffusion coefficient.

\section{Results and Discussion}

To assess the suitability of the instrumentation for a space experiment, tests of the developed breadboard have been performed. These tests were aimed both at the evaluation of the thermal and optical performances of the breadboard, and at the

verification of the suitability of the breadboard to perform scientific measurements on non-equilibrium fluctuations in binary and ternary mixtures under conditions similar to those envisioned for the space experiments of the Giant Fluctuations project. The main goal of the scientific tests was to determine the static structure factor of fluctuations to assess whether its signal to noise ratio at small wave vectors is at least of the order of ten for the largest temperature differences that can be applied to the sample. This condition is required for a reliable determination of the structure factor of small-amplitude nonequilibrium concentration fluctuations.

\section{Thermal Performances of the Breadboard Cell}

One of the most important parameters for the interpretation of the scientific results that will be collected during the flight is the effective value of the temperature difference $\Delta T_{e}$ applied to the sample. Although simulations and experimental calibrations allow to determine $\Delta T_{e}$ from the temperature difference at the surface of the Peltier elements, these methods provide sample-specific results. Moreover, tests measurements performed with toluene show that the relative temperature drop measured by thermistors depends on the magnitude of the applied temperature difference (typical values are shown in Table 2).

For these reasons, thin film thermistors have been integrated on the inner surfaces of the sapphire windows. The integration of thin film thermistors allows to measure directly the actual temperature difference $\Delta T_{e}$. In order to assess the reliability of the measurement of the temperature difference applied to the sample obtained with thin film thermistors, we estimated the threshold of the Rayleigh-Bénard instability triggered by heating a sample of pure toluene from below. According to the theory of convective instabilities, the critical Rayleigh number needed for the onset of the Rayleigh-Bénard instability is $R_{c}=1708$ for a layer of fluid of high aspect ratio, and this corresponds to a critical temperature difference $\Delta T_{c}=-9.01 \mathrm{~K}$ for a $1.00 \mathrm{~mm}$ thick layer of toluene.

The threshold temperature needed to trigger a RayleighBénard convective instability was estimated by imposing an increasing (in modulus) temperature difference to the toluene sample. A temperature difference $\Delta T_{e}=-9.08 \mathrm{~K}$, as measured by the thin film thermistors, resulted in no convection, while a temperature difference $\Delta T_{e}=-9.5 \mathrm{~K}$ led to the development of convection rolls (Fig. 5). These measurements

Table 2 Temperature difference at the inner and outer surface of sapphire windows

\begin{tabular}{lll}
\hline$\Delta T$ at Peltier & $\Delta T_{e}$ at sample & $\%$ drop \\
\hline $5 \mathrm{~K}$ & $3.55 \mathrm{~K}$ & $29 \%$ \\
$10 \mathrm{~K}$ & $7.9 \mathrm{~K}$ & $21 \%$ \\
$20 \mathrm{~K}$ & $16.6 \mathrm{~K}$ & $17 \%$ \\
\hline
\end{tabular}


provided an indication that the threshold temperature difference for the onset of Rayleigh-Bénard convection falls in-between these two values. This result is compatible with the theoretical prediction for the threshold of the instability $\Delta T_{c}=$ $-9.01 \mathrm{~K}$, within the error in the determination of the theoretical value of the threshold. The outcome of the tests on the performances of the thin film thermistors is that they provide a fast and reliable solution for an accurate determination of the temperature difference imposed to the sample, without the need of sample-dependent calibrations.

\section{Lensing and Radial Gradient}

The presence of a radial temperature gradient inside the sample leads to a focusing/defocusing of the beam of light impinging on the sample. Currently, the only available theoretical modelling for the lensing effect is the one developed for the self-focusing of a beam of light during the propagation in an absorbing medium, an effect known as thermal lensing. A careful analysis of the thermal lensing effect showed that the absence of a strong longitudinal gradient makes it dramatically different from the case where such a longitudinal gradient is present, as it occurs in all the Giant Fluctuations experiments. For this reason, the theoretical description developed for thermal lensing is not suitable to model the lensing effect generated by a radial gradient in the Giant Fluctuations breadboard cell. Therefore, we developed an ad-hoc model, suitable for the characterization of the possible lensing effect generated by radial gradients in the breadboard cell. This model allows to determine the radial temperature profile $T_{0}(r)$ at the midheight of the cell which, under ideal conditions, should be perfectly flat.

We outline below the main results of this model:

- The sample has a cylindrical shape, with radius $R$ and height $h$. A temperature distribution $T(r, z)$ is present inside the sample, where $0<r<R$ is the radial coordinate and $-h /$ $2<z<h / 2$ the longitudinal one (parallel to the temperature gradient). The temperature difference $T(r, z)-T_{0}(r)$ is
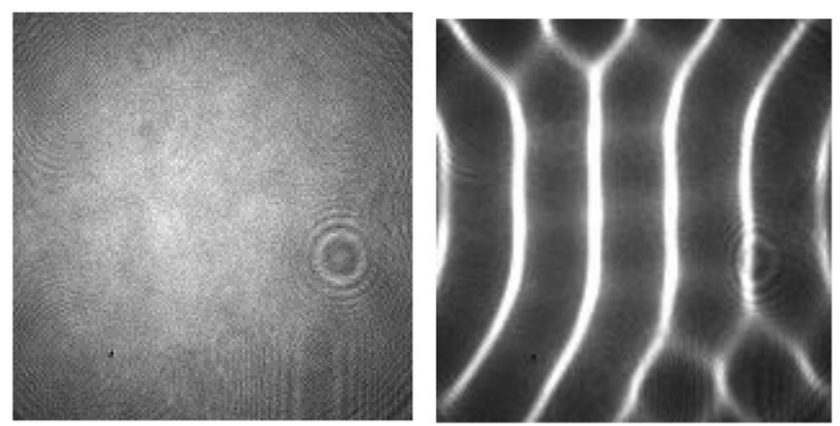

Fig. 5 Toluene sample under the action of a temperature difference, heated from below to induce a Rayleigh-Bénard convective instability; left, $\Delta T_{e}=-9.08 \mathrm{~K}$ (no convection); right, $\Delta T_{e}=-9.5 \mathrm{~K}$ (convection)

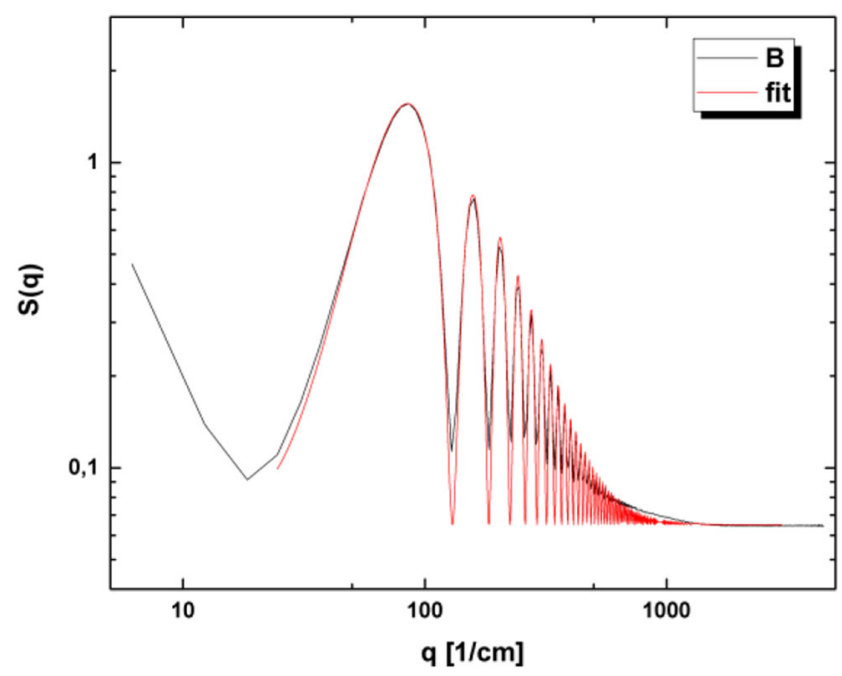

Fig. 6 Static power spectrum of a sequence of shadowgraph images collected during thermodiffusion in binary mixture for $\Delta T=20 \mathrm{~K}$. The red line is the best fit by using available theoretical models

assumed to be anti-symmetric with respect to $z$. Here $T_{0}(r)=T(r, 0)$ is the temperature at the mid-height of the cell. Under these conditions the optical path travelled by a beam of light crossing the sample along $\mathrm{z}$ is independent on $z$ and can be written as:

$l(r)=n_{0} h+\frac{d n}{d T}\left[T_{0}(r)-T_{0}\right] h$,

where $T_{0}$ and $n_{0}$ are respectively the temperature and the refractive index at the centre of the cell $(r=0, z=0)$.

- The lensing effect determined by the radial temperature profile gives rise to a relative variation of the diameter of a beam of light given by:

$\frac{D-D^{\prime}}{D}=\frac{2 h\left[T_{0}(R)-T_{0}\right] Z}{R^{2}} \frac{d n}{d T}$

where $D$ and $D^{\prime}$ are the diameters of the beam at a distance $\mathrm{Z}$ from the sample in the absence and in the presence of a temperature gradient imposed to the sample, respectively.

The maximum tolerable radial temperature variation is $1 \%$ of the imposed temperature difference $\Delta T$ expressed in degrees Celsius. Under worst-case conditions, $\Delta T=20 \mathrm{~K}$, $T_{0}(R)-T_{0}=0.2 \mathrm{~K}, R=8 \mathrm{~mm}$ and $d n / d t=-5.6 \times 10^{-4} \mathrm{~K}^{-1}$. Therefore, the maximum tolerable variation of the beam diameter determined by the lensing effect is $\left(D-D^{\prime}\right) / D=1.3 \times$ $10^{-3}$.

In order to determine experimentally the relative change of the beam diameter, two series of 1000 full-frame images were 
collected at $12.5 \mathrm{~Hz}$ with the Continuous Wave (CW) red light source. One sequence was collected in the presence of a temperature difference $\Delta T=20 \mathrm{~K}$ and the other in the absence of the latter. For each one of the two temperature differences, the beam diameter was obtained by averaging a sequence of 100 images to reduce noise, by applying an arbitrary threshold to the averaged images, and by determining the area and average diameter of the binarized beam profile.

This procedure resulted in a relative variation of the beam diameter of the order of $\left(D-D^{\prime}\right) / D=2.5 \times 10^{-4}$ or smaller, fully compatible with the requirement that the variation must be smaller than $1.3 \times 10^{-3}$.

\section{Calibration of Wave Vectors}

The CCD sensor used during the tests is the AVT Prosilica GT2300, which includes a detector with $2336 \times 1752$ pixels of size of $5.5 \mu \mathrm{m} /$ pixel. For the analysis of non-equilibrium fluctuations (NEFs), images of $1024 \times 1024$ pixels are used. The size of the detector for such number of pixels is then 1024 pixels $=$ $5.63 \mathrm{~mm}$. To know the size of the image in the real space one needs to know the optical magnification that is set from the focal distance of the lens and the distance between the lens and the sample. For the magnification $\mathrm{M}=0.55$ adopted in the setup it follows $\mathrm{L}=$ $10.24 \mathrm{~mm}$ for the region of interest (ROI) of $1024 \times$ 1024 pixels and thus a $q_{\min }=2 \pi / \mathrm{L}=6.14 / \mathrm{cm}$.

\section{Structure Factor of Non-equilibrium Fluctuations in a Binary Mixture}

Scientific tests required to determine the signal to noise ratio of the structure factor of non-equilibrium fluctuations occurring during a thermodiffusion process under conditions similar to those that will be adopted in space experiments. A first investigated sample was a diluted polystyrene polymer solution binary mixture (Toluene $98 \%+$ PS 2\%) with the same molecular weight and concentration of the one used in GRADFLEX.

Tests were performed according to the following procedure. The cell was filled and levelled accurately, and its temperature was let stabilize at $T=25{ }^{\circ} \mathrm{C}$ for $1 \mathrm{~h}$. Two sequences of 1000 images each were acquired at steady state at $25 \mathrm{~Hz}$ and at $2.5 \mathrm{~Hz}$ by using the $\mathrm{CW}$ red and violet sources. A temperature difference $\Delta T=10 \mathrm{~K}$ was then imposed to the sample by heating from above. After $1 \mathrm{~h}$, when stationary conditions were reached, two sequences of 1000 images at $25 \mathrm{~Hz}$ and at $2.5 \mathrm{~Hz}$ were acquired by using the CW red source and $\mathrm{CW}$ violet source. The same procedure was repeated for a temperature difference $\Delta T=20 \mathrm{~K}$.

This test was aimed at assessing the overall quality of the images, and in particular the camera noise, and the amplitude of the signal generated by non-equilibrium fluctuations. For each of the two applied temperature differences $(\Delta T=10 \mathrm{~K}$ and $\Delta T=20 \mathrm{~K}$ ) and for each of the two illumination condition (CW violet source, $\lambda=405 \mathrm{~nm}$ and $\mathrm{CW}$ red source, $\lambda=$ $650 \mathrm{~nm})$, the images were processed with a Differential Dynamic Analysis DDA algorithm. Two image sequences, each of 1000 shadowgraph images, taken at the nominal frame rate of 25 frames/s, and 2.5 frames/s, respectively, were analyzed to obtain the azimuthally averaged image structure function $D(q, \Delta t)$ spanning three orders of magnitude in time.

As shown in Fig. 7, the ISF is well fitted to a double exponential function:

$$
\begin{aligned}
D(q, \Delta t)= & A_{f}(q)\left(1-e^{-\Gamma_{f}(\mathrm{q}) \Delta t}\right)+A_{s}(q)\left(1-e^{-\Gamma_{s}(\mathrm{q}) \Delta t}\right) \\
& +B(q)
\end{aligned}
$$

where $A_{f}\left(A_{s}\right)$ and $\Gamma_{f}\left(\Gamma_{s}\right)$ are the amplitudes and the decay rates associated with the fast (slow) decay, respectively, and $B$ is a baseline term due to the noise in the detection chain. We attribute the fast (slow) decay to the relaxation of temperature (concentration) non-equilibrium fluctuations. We note that a reliable estimate of the fast decay rate can be obtained as far as $\Gamma_{f}(\mathrm{q})$ is below the frame rate, i.e. for $q \leq 210^{4} \mathrm{~m}^{-1}$.

At small wave vectors the decay rates are significantly affected by the presence of the terrestrial gravity force, and the relaxation of fluctuations is determined by buoyancy (Segré and Sengers 1993; Vailati and Giglio 1996; Ortiz de Zárate and Sengers 2006). Conversely, at large wave vectors the relaxation of temperature and concentration fluctuations occurs by conduction and diffusion, respectively. Under these conditions, the fast and slow relaxation rates are $\Gamma_{f}(q)=K q^{2}$ and $\Gamma_{s}(q)=D q^{2}$, where $K$ is the thermal diffusivity. By using these espressions for the decay rates to fit the structure functions at large wave vector we get $K_{\text {est }}=(0.9 \pm 0.2) \times 10^{-7} \mathrm{~m}^{2} / \mathrm{s}$ and $D_{\text {est }}=(2.2 \pm 0.2) \times$

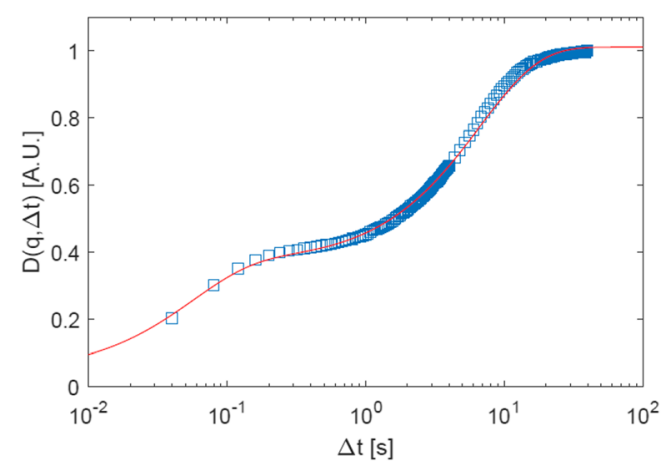

Fig. 7 Representative image structure function for $q=1.510^{4} \mathrm{~m}^{-1}, \Delta T=$ $10 \mathrm{~K}$ and $\lambda=405 \mathrm{~nm}$ obtained from DDA analyisis of the shadographic images of the diluted polymer binary mixture 
$10^{-10} \mathrm{~m}^{2} / \mathrm{s}$, in good agreement with the reference values $K=0.89 \times 10^{-7} \mathrm{~m}^{2} / \mathrm{s}$ (Li et al. 1994) and $D=1.83 \times$ $10^{-10} \mathrm{~m}^{2} / \mathrm{s}$ (Zhang et al. 1999).

An estimate of the overall signal to noise ratio can be obtained by considering the quantity $\left(A_{f}+A_{s}\right) / B$, which is represented in Fig. 8, for all the considered cases. As it can be appreciated from the figure, the height of the first peak ranges from about 7 (for the largest wavelength and the lower temperature gradient) to about 32 (for the shorter wavelength and the higher temperature gradient). Considering only the higher applied temperature gradient, the normalized amplitude of the first peak is above 10 for both sources and testifies a low level of noise fully suitable to perform experiments under microgravity conditions.

\section{Structure Factor of Non-equilibrium Fluctuations in a Diluted Ternary Mixture}

Tests have been performed on the ternary mixture (PS $2 \%$ + toluene $39 \%$ + cyclohexane $59 \%$ ) by using a procedure mirroring that adopted for the binary mixture. For a temperature difference $\Delta T=20 \mathrm{~K}$ and for each of the two illumination condition ( $\mathrm{CW}$ violet source, $\lambda=$ $405 \mathrm{~nm}$ and $\mathrm{CW}$ red source, $\lambda=650 \mathrm{~nm}$ ), the images
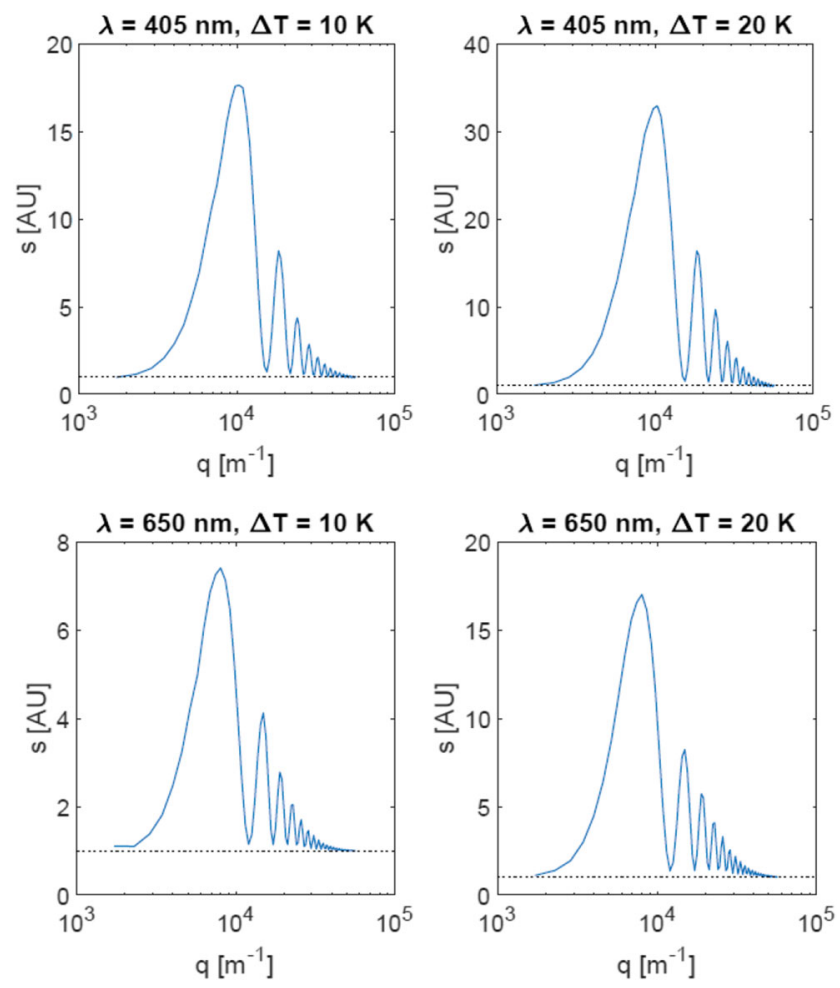

Fig. 8 Signal to noise ratio of the optical signal of the binary mixture as a function of the wave vector $\mathbf{q}$, normalized by the noise baseline. The first line corresponds to the violet light source, while the second to the red one. The first column corresponds to an imposed temperature difference $\Delta T=$ $10 \mathrm{~K}$, while the second to $\Delta T=20 \mathrm{~K}$ where processed with a Differential Dynamic Analysis DDA algorithm. Two image sequences, each of 1000 shadowgraph images, taken at the nominal frame rate of 25 frames/s and 2.5 frames/s, respectively, were analysed to obtain the image structure function (ISF) $D(q, \Delta t)$. An example, shown in Fig. 9, shows that the ISF is well fitted to a double exponential function for most wave vectors.

The amplitude of the images has been evaluated as described above for the binary mixture. An equivalent normalisation has also been applied. As it can be appreciated from the Fig. 10, the height of the first peak ranges from about 16.4 (for the largest wavelength) to about 37.4 (for the shorter wavelength). The normalized amplitude of the first peak is above 10 for both sources and the experimental system is suitable for the characterization of non-equilibrium fluctuations in space occurring in a ternary mixture where one of the components is polymer at a dilute concentration.

\section{Structure Factor of Non-equilibrium Fluctuations in a Concentrated Ternary Mixture}

Tests have been performed on the ternary mixture (PS $50 \%+$ toluene $25 \%$ + cyclohexane $25 \%$ ) by using a procedure mirroring the one that adopted for the binary mixture (see Fig. 11). These tests were aimed at assessing the performances of the experimental system during the investigation of non-equilibrium fluctuations occurring in a ternary mixture including a concentrated polymer, under conditions of interest for the investigation of non-equilibrium fluctuations close to a glass transition. The structure function reported in Fig. 11 is qualitatively quite different from those shown in Figs. 7

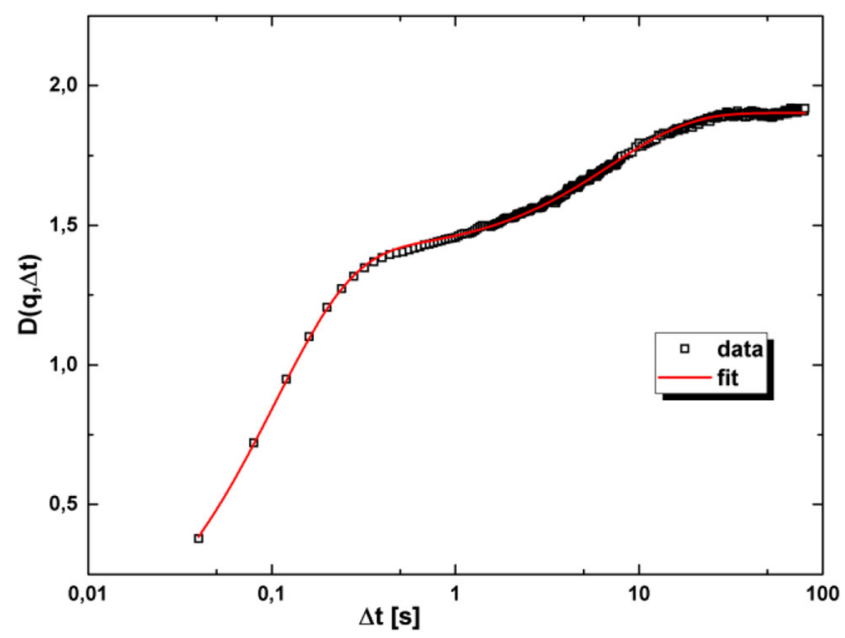

Fig. 9 Representative image structure function $\mathrm{D}(\mathrm{q}, \Delta \mathrm{t})$, corresponding to $\mathrm{q}=9.210^{3} \mathrm{~m}^{-1}, \Delta T=20 \mathrm{~K}$ and $\lambda=405 \mathrm{~nm}$, obtained from DDA analysis of the shadowgraphic images of the diluted ternary mixture 

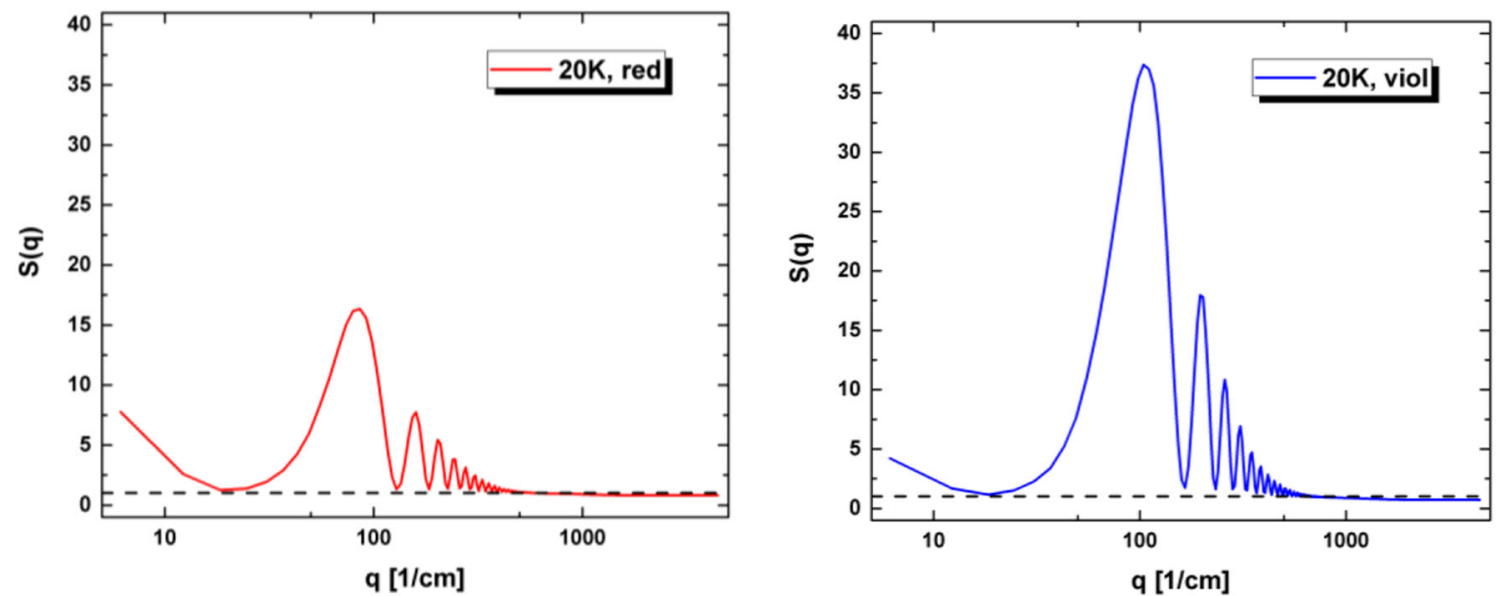

Fig. 10 Signal to noise ratio of the optical signal of the ternary mixture of diluted PS in toluene and cyclohexane, plotted as a function of the wave vector q. The curve is normalized by the noise baseline. The left column corresponds to the red light source, while the right column to the violet source

and 9. The main point is the lack of an evident exponential decay behaviour. On the contrary, a steep increase of the structure function can be detected as a function of the delay time $\Delta t$, probably related to the non-stationary state of the concentrated polymeric mixture. The peculiar shape of the structure function is, indeed, an indication that the situation in dense polymeric solutions can be much more complex than in simple liquids. The investigation of such systems is one of the topics that shall be addressed during the Neuf-Dix project. The strange behaviour of the structure function for very large delay times around $50 \mathrm{~s}$ is probably related to noise of the specific measurement. A more detailed analysis of the shown structure function is outside the scope of the present paper and will be performed in future publications.

The static structure factor of the images has been evaluated as described above for the binary mixture. An equivalent normalisation has also been applied. As it can be appreciated from Fig. 12, the height of the first peak ranges from about 72 (for the largest wavelength) to about 228 (for the shorter wavelength). The normalized amplitude of the first peak is well above 10 for both sources. The fact that the signal to noise ratio for this sample largely exceeds the threshold indicates that the developed experimental setup can be used to characterize very accurately the nonequilibrium fluctuations occurring during
Fig. 11 Representative image structure function $D(q, \Delta t)$, corresponding to $q=9.210^{3} \mathrm{~m}^{-1}$, $\Delta T=20 \mathrm{~K}$ and $\lambda=405 \mathrm{~nm}$, obtained from DDA analysis of the shadowgraphic images of the ternary mixture of concentrated PS in toluene and cyclohexane

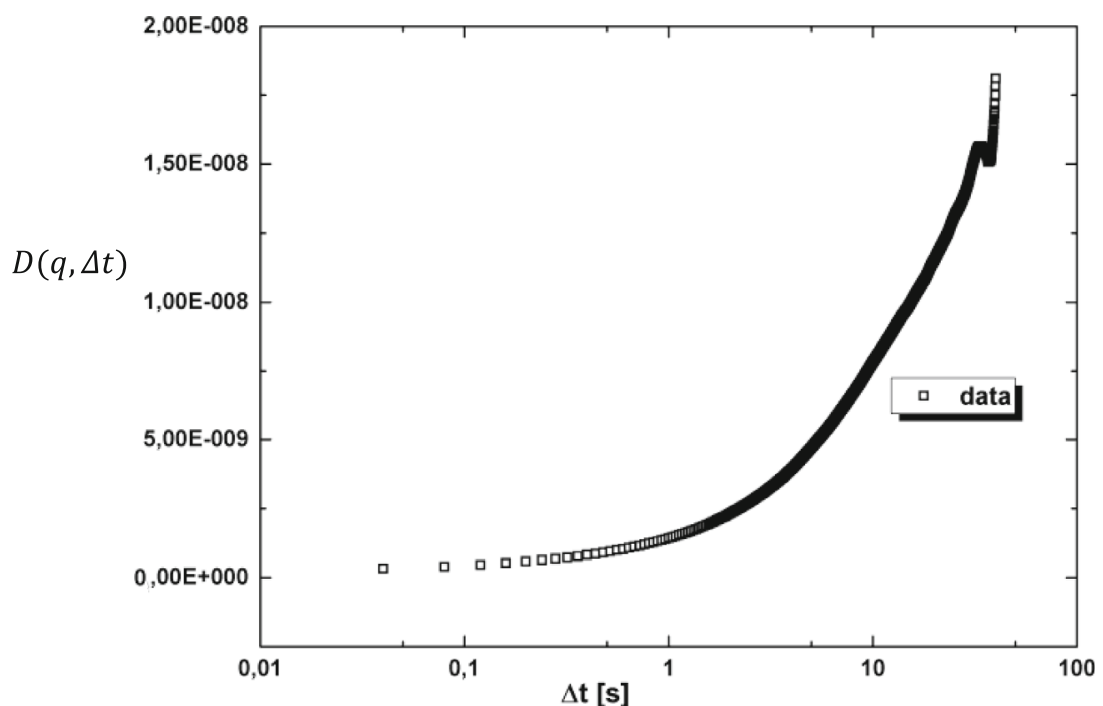



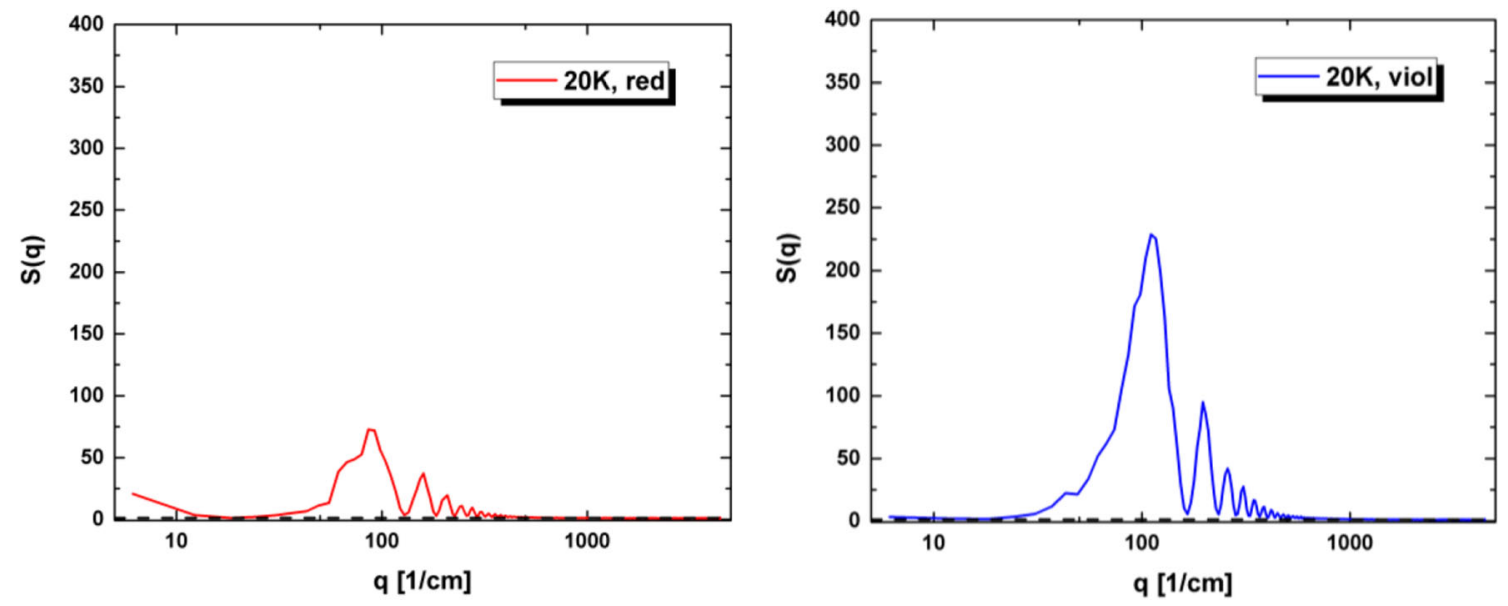

Fig. 12 Signal to noise ratio of the optical signal for the ternary mixture of concentrated PS in toluene and cyclohexane, plotted as a function of the wave vector q. The curve is normalized by the noise baseline. The left column corresponds to the red light source, while the right one to the violet source

thermodiffusion in a ternary mixture where one of the components is a concentrated polymer.

\section{Conclusions}

Tests of the Giant Fluctuations experimental breadboard performed at the end of the phase $\mathrm{A} / \mathrm{B}$ allowed to confirm that its thermal and optical performances are suitable for the scientific characterization of the static and dynamic structure factors of non-equilibrium fluctuations in space. The diagnostics includes two light sources operating at different wavelengths of $405 \mathrm{~nm}$ and $650 \mathrm{~nm}$. Further improvements will consist in the optimization of the triggering of the light sources, so to be able to collect shadowgraph images at 25 frames per second by rapidly alternating one acquisition with the red source to another with the violet one. Preliminary tests have shown that optical switching of the light sources by means of an opto-mechanical actuator allows to achieve this purpose, and further improvements will be devoted to the optimization of the synchronism between the triggering of the source and of the camera.

Acknowledgements We acknowledge support from the European Space Agency (ESA). FC, HB, and LGF acknowledge support from the French Centre Nationale d'Etudes Spatiales (CNES) and from Excellence Initiative of University of Pau and Countries of Adour - I-Site E2S, a French "Investissements d'Avenir" programme. WK and DZ acknowledge support from the Deutsches Zentrum für Luft- und Raumfahrt (DLR grant 50WM1850).

Funding Information Open access funding provided by Università degli Studi di Milano within the CRUI-CARE Agreement.

Open Access This article is licensed under a Creative Commons Attribution 4.0 International License, which permits use, sharing, adaptation, distribution and reproduction in any medium or format, as long as you give appropriate credit to the original author(s) and the source, provide a link to the Creative Commons licence, and indicate if changes were made. The images or other third party material in this article are included in the article's Creative Commons licence, unless indicated otherwise in a credit line to the material. If material is not included in the article's Creative Commons licence and your intended use is not permitted by statutory regulation or exceeds the permitted use, you will need to obtain permission directly from the copyright holder. To view a copy of this licence, visit http://creativecommons.org/licenses/by/4.0/.

\section{References}

Baaske, P., Bataller, H., Braibanti, M., Carpineti, M., Cerbino, R., Croccolo, F., Donev, A., Köhler, W., Ortiz de Zárate, J.M., Vailati, A.: The NEUF-DIX space project - non-equilibrium fluctuations during diffusion in complex liquids. Eur. Phys. J. E. 39, 119 (2016)

Balboa Usabiaga, F., et al.: Staggered schemes for fluctuating hydrodynamics. SIAM J. Multiscale Model. Simul. 10, 1369-1408 (2012)

Bataller, H., Giraudet, C., Croccolo, F., Ortiz de Zárate, J.M.: Analysis of non-equilibrium fluctuations in a ternary liquid mixture. Microgravity Sci. Technol. 28, 611 (2016)

Bataller, H., Triller, T., Pur, B., Köhler, W., Ortiz de Zárate, J.M., Croccolo, F.: Dynamic analysis of the light scattered by the nonequilibrium fluctuations of a ternary mixture of polystyrene-toluenen-hexane. Eur. Phys. J. E. 40, 35 (2017)

Bou-Ali, M.M., Ahadi, A., Alonso de Mezquia, D., Galand, Q., Gebhardt, M., Khlybov, O., Köhler, W., Larrañaga, M., Legros, J.C., Lyubimova, T., Mialdun, A., Ryzhkov, I., Saghir, M.Z., Shevtsova, V., Van Vaerenbergh, S.: Benchmark values for the Soret, thermodiffusion and molecular diffusion coefficients of the ternary mixture tetralin+isobutylbenzene+n-dodecane with $0.8-0.1-$ 0.1 mass fraction. Eur. Phys. J. E. 38, 30 (2015)

Braibanti, M., Artola, P.A., Baaske, P., Bataller, H., Bazile, J.P., Bou-Ali, M.M., Cannell, D.S., Carpineti, M., Cerbino, R., Croccolo, F., Diaz, J., Donev, A., Errarte, A., Ezquerro, J.M., Frutos-Pastor, A., Galand, Q., Galliero, G., Gaponenko, Y., García-Fernández, L., Gavaldá, J., Giavazzi, F., Giglio, M., Giraudet, C., Hoang, H., Kufner, E., Köhler, W., Lapeira, E., Laverón-Simavilla, A., Legros, J.C., Lizarraga, I., Lyubimova, T., Mazzoni, S., Melville, N., Mialdun, A., Minster, O., 
Montel, F., Molster, F.J., Ortiz de Zárate, J.M., Rodríguez, J., Rousseau, B., Ruiz, J., Ryzhkov, I.I., Schraml, M., Shevtsova, V., Takacs, C.J., Triller, T., Van Vaerenbergh, S., Vailati, A., Verga, A., Vermorel, R., Vesovic, V., Yasnou, V., Xu, S., Zapf, D., Zhang, K.: European Space Agency experiments on thermodiffusion of fluid mixtures in space. Eur. Phys. J. 42, 86 (2019)

Brogioli, D., Vailati, A.: Nonequilibrium fluctuations during diffusion in liquid layers. Phys. Rev. E. 96, 012136 (2017)

Brogioli, D., Vailati, A., Giglio, M.: Universal behavior of nonequilibrium fluctuations in free diffusion processes. Phys. Rev. E. 61, R1-R4 (2000)

Brogioli, D., Croccolo, F., Vailati, A.: Correlations and scaling properties of non-equilibrium fluctuations in liquid mixtures. Phys. Rev. E. 94, 022142 (2016)

Brogioli, D., Croccolo, F., Vailati, A.: Asymmetric time-cross-correlation of nonequilibrium concentration fluctuations in a ternary liquid mixture. Phys. Rev. E. 99, 053115 (2019)

Carpineti, M., Sabato, M., Croccolo, F., Vailati, A.: Kinetics of growth of non-equilibrium fluctuations during thermodiffusion in a polymer solution. Eur. Phys. J. E. 42, 25 (2019)

Cerbino, R., Trappe, V.: Differential dynamic microscopy: probing wave vector dependent dynamics with a microscope. Phys. Rev. Lett. 100, 188102 (2008)

Cerbino, R., Vailati, A.: Near-field scattering techniques: novel instrumentation and results from time and spatially resolved investigations of soft matter systems. Curr. Opin. Colloid Interface Sci. 14, 416 425 (2009)

Cerbino, R., Sun, Y., Donev, A., Vailati, A.: Dynamic scaling for the growth of non-equilibrium fluctuations during thermophoretic diffusion in microgravity. Sci. Rep. 5, 14486 (2015)

Croccolo, F., Brogioli, D.: Quantitative Fourier analysis of Schlieren masks: the transition from shadowgraph to Schlieren. App. Opt. 50, 3419 (2011)

Croccolo, F., Brogioli, D., Vailati, A., Giglio, M., Cannell, D.S.: Use of dynamic Schlieren interferometry to study fluctuations during free diffusion. App. Opt. 45, 2166 (2006)

Croccolo, F., Brogioli, D., Vailati, A., Giglio, M., Cannell, D.S.: Nondiffusive decay of gradient-driven fluctuations in a freediffusion process. Phys. Rev. E. 76, 041112 (2007)

Croccolo, F., Bataller, H., Scheffold, F.: A light scattering study of non equilibrium fluctuations in liquid mixtures to measure the Soret and mass diffusion coefficient. J. Chem. Phys. 137, 234202 (2012)

Croccolo, F., Giraudet, C., Bataller, H., Cerbino, R., Vailati, A.: Shadowgraph analysis of non-equilibrium fluctuations for measuring transport properties in microgravity in the GRADFLEX experiment. Microgravity Sci. Technol. 28, 467-475 (2016)

Croccolo, F., Garcia-Fernández, L., Bataller, H., Vailati, A., Ortiz de Zárate, J. M.: Propagating modes in a binary liquid mixture under thermal stress, Phys. Rev. E 99, 012602 (2019)

Delong, S., Sun, Y., Griffith, B.E., Vanden-Eijnden, E., Donev, A.: Multiscale temporal integrators for fluctuating hydrodynamics. Phys. Rev. E. 90, 063312 (2014)

Donev, A., de la Fuente, A., Bell, J.B., Garcia, A.L.: Diffusive transport enhanced by thermal velocity fluctuations. Phys. Rev. Lett. 106, 204501 (2011)

Donev, A., Fai, T.G., Vanden-Eijnden, E.: A reversible mesoscopic model of diffusion in liquids: from giant fluctuations to Fick's law. J. Stat. Mech. P04004, 1-39 (2014a)

Donev, A., Vanden-Eijnden, E.: Dynamic density functional theory with hydrodynamic interactions and fluctuations. J. Chem. Phys. 140, 234115 (2014b)

Enge, W., Köhler, W.: Thermal diffusion in a critical polymer blend. Phys. Chem. Chem. Phys. 6, 2373-2378 (2004)

Galand, Q., Van Vaerenbergh, S., Köhler, W., Khlybov, O., Lyubimova, T., Mialdun, A., Ryzhkov, I., Shevtsova, V., Triller, T.: Results of the DCMIX1 experiment on measurement of Soret coefficients in ternary mixtures of hydrocarbons under microgravity conditions on the ISS. J. Chem. Phys. 151, 134502 (2019)

Giavazzi, F., Cerbino, R.: Digital Fourier microscopy for soft matter dynamics. J. Opt. 16, 083001 (2014)

Giavazzi, F., Savorana, G., Vailati, A., Cerbino, R.: Structure and dynamics of concentration fluctuations in a non-equilibrium dense colloidal suspension. Soft Matter. 12, 6558 (2016a)

Giavazzi, F., Fornasieri, A., Vailati, A., Cerbino, R.: Equilibrium and non-equilibrium concentration fluctuations in a critical binary mixture. Eur. Phys. J. E. 39, 103 (2016b)

Giraudet, C., Bataller, H., Croccolo, F.: High-pressure mass transport properties measured by dynamic near-field scattering of nonequilibrium fluctuations. Eur. Phys. J. E. 37, 107 (2014)

Giraudet, C., Bataller, H., Sun, Y., Donev, A., Ortiz de Zárate, J.M., Croccolo, F.: Slowing-down of non-equilibrium concentration fluctuations in confinement. Europhys. Lett. 111, 60013 (2015)

de Groot, S.R., Mazur, P.: Nonequilibrium Thermodynamics. Dover, New York (1984)

Kirkpatrick, T.R., Ortiz de Zárate, J.M., Sengers, J.V.: Giant Casimir effect in fluids in nonequilibrium steady states. Phys. Rev. Lett. 110, 235902 (2013)

Kirkpatrick, T.R., Ortiz de Zárate, J.M., Sengers, J.V.: Nonequilibrium Casimirlike forces in liquid mixtures. Phys. Rev. Lett. 115, 035901 (2015)

Li, W.B., Segrè, P.N., Gammon, R.W., Sengers, J.V.: Small-angle Rayleigh scattering from nonequilibrium fluctuations in liquids and liquid mixtures. Physica A. 204, 399-436 (1994)

Lizarraga, I., Giraudet, C., Croccolo, F., Bou-Ali, M.M., Bataller, H.: Mass diffusion and thermal diffusivity of the decane-pentane mixture under high pressure as a ground-based study for SCCO project. Microgravity Sci. Technol. 28, 267 (2016)

Mialdun, A., Bataller, H., Bou-Ali, M.M., Braibanti, M., Croccolo, F., Errarte, A., Ezquerro, J.M., Gaponenko, Y., Garcia-Fernàndez, L., Fernàndez, J.J., Rodriguez, J., Shevtsova, V.: Preliminary analysis of diffusion coefficient measurements in ternary mIXtures 4 (DCMIX4) experiment on-board the international Space Station. Eur. Phys. J. E. 42, 87 (2019)

Oprisan, A., Oprisan, S., Teklu, A.: Experimental study of nonequilibrium fluctuations during free diffusion in nanocolloids using microscopic techniques. Appl. Opt. 49, 86-98 (2010)

Ortiz de Zárate, J.M., Sengers, J.V.: Hydrodynamic Fluctuations. Elsevier, Amsterdam (2006)

Ortiz de Zárate, J.M., Fornés, J.A., Sengers, J.V.: Long-wavelength nonequilibrium concentration fluctuations induced by the Soret effect. Phys. Rev. E. 74, 046305 (2006)

Ortiz de Zárate, J.M., Kirkpatrick, T.R., Sengers, J.V.: Non-equilibrium concentration fluctuations in binary liquids with realistic boundary conditions. Eur. Phys. J. E. 38, 99 (2015)

Rauch, J., Köhler, W.: Diffusion and thermal diffusion of semidilute to concentrated solutions of polystyrene in toluene in the vicinity of the glass transition. Phys. Rev. Lett. 88, 185901 (2002)

Rauch, J., Köhler, W.: Collective and thermal diffusion in dilute, semidilute, and concentrated solutions of polystyrene in toluene. J. Chem. Phys. 119, 11977-11988 (2003)

Schmitz, R.: Fluctuations in a nonequilibrium colloidal suspension. Physica A. 206, 25-57 (1994)

Segrè, P.N., Sengers, J.V.: Nonequilibrium fluctuations in liquid mixtures under the influence of gravity. Physica A. 198, 46-77 (1993)

Šeta, B., Errarte, A., Dubert, D., Gavaldà, J., Bou-Ali, M.M., Ruiz, X. Gravitational stability analysis on double diffusion convection in ternary mixtures. Acta Astronaut. 160, 442-450 (2019)

Shevtsova, V., Sechenyh, V., Nepomnyashchy, A., Legros, J.C.: Analysis of the application of optical two-wavelength techniques to measurement of the Soret coefficients in ternary mixtures. Philos. Mag. 91, 3498-3518 (2011) 
Shevtsova, V., et al.: Diffusion and soret in ternary mixtures. Preparation of the DCMIX2 experiment on the ISS. Microgravity Sci. Tec. 25, 275-283 (2014)

Takacs, C.J., Vailati, A., Cerbino, R., Mazzoni, S., Giglio, M., Cannell, D.S.: Thermal fluctuations in a layer of CS2 subjected to temperature gradients with and without the influence of gravity. Phys. Rev. Lett. 106, 244502 (2011)

Trainoff, S., Cannell, D.S.: Physical optics treatment of the shadowgraph. Phys. Fluids. 14, 1340-1363 (2002)

Triller, T., Bataller, H., Bou-Ali, M.M., Braibanti, M., Croccolo, F., Ezquerro, J.M., Galand, Q., Gavalda, J., Lapeira, E., LaveronSimavilla, A., Lyubimova, T., Mialdun, A., Ortiz de Zárate, J.M., Rodriguez, J., Ruiz, X., Ryzhkov, I.I., Shevtsova, V., Van Vaerenbergh, S., Köhler, W.: Thermodiffusion in ternary mixtures of water/ethanol/triethylene glycol: first report on the DCMIX3experiments performed on the International Space Station. Microgravity Sci. Tec. 30, 295 (2018)

Vailati, A., Giglio, M.: Q divergence of nonequilibrium fluctuations and its gravity induced frustration in a temperature stressed liquid mixture. Phys. Rev Lett. 77, 1484 (1996)
Vailati, A., Giglio, M.: Giant fluctuations in a free diffusion process. Nature. 390, 262-265 (1997)

Vailati, A., Giglio, M.: Nonequilibrium fluctuations in time-dependent diffusion processes. Phys. Rev. E. 58, 4361-4371 (1998)

Vailati, A., Cerbino, R., Mazzoni, S., Giglio, M., Nikolaenko, G., Takacs, C.J., Cannell, D.S., Meyer, W.V., Smart, A.E.: Gradient-driven fluctuations experiment: fluid fluctuations in microgravity. App. Opt. 45, 2155-2165 (2006)

Vailati, A., Cerbino, R., Mazzoni, S., Takacs, C.J., Cannell, D.S., Giglio, M.: Fractal fronts of diffusion in microgravity. Nature Comm. 2, 290 (2011)

Zhang, K.J., Briggs, M.E., Gammon, R.W., Sengers, J.V.: Thermal and mass diffusion in a semidilute good solvent-polymer solution. J. Chem. Phys. 111, 2270-2282 (1999)

Publisher's Note Springer Nature remains neutral with regard to jurisdictional claims in published maps and institutional affiliations. 